\title{
Whole Earth Telescope observations of BPM 37093: A seismological test of crystallization theory in white dwarfs ${ }^{\star}$
}

A. Kanaan ${ }^{1}$, A. Nitta ${ }^{2}$, D. E. Winget ${ }^{3}$, S. O. Kepler ${ }^{4}$, M. H. Montgomery ${ }^{5,3}$, T. S. Metcalfe ${ }^{6,3}$, H. Oliveira $^{1}$, L. Fraga ${ }^{1}$, A. F. M. da Costa ${ }^{4}$, J. E. S. Costa ${ }^{4}$, B. G. Castanheira ${ }^{4}$, O. Giovannini ${ }^{7}$, R. E. Nather ${ }^{3}$, A. Mukadam ${ }^{3}$, S. D. Kawaler ${ }^{8}$, M. S. O’Brien ${ }^{8}$, M. D. Reed ${ }^{8,9}$, S. J. Kleinman' ${ }^{2}$, J. L. Provencal ${ }^{10}$, T. K. Watson ${ }^{11}$, D. Kilkenny ${ }^{12}$, D. J. Sullivan ${ }^{13}$, T. Sullivan ${ }^{13}$, B. Shobbrook ${ }^{14}$, X. J. Jiang ${ }^{15}$, B. N. Ashoka ${ }^{16}$, S. Seetha ${ }^{16}$, E. Leibowitz ${ }^{17}$, P. Ibbetson ${ }^{17}$, H. Mendelson ${ }^{17}$, E. G. Meištas ${ }^{18}$, R. Kalytis ${ }^{18}$, D. Ališauskas ${ }^{19}$, D. O’Donoghue ${ }^{12}$, D. Buckley ${ }^{12}$, P. Martinez ${ }^{12}$, F. van Wyk ${ }^{12}$, R. Stobie ${ }^{12}$, F. Marang ${ }^{12}$, L. van Zyl ${ }^{12}$, W. Ogloza ${ }^{20}$, J. Krzesinski ${ }^{20}$, S. Zola ${ }^{20,21}$, P. Moskalik ${ }^{22}$, M. Breger ${ }^{23}$, A. Stankov ${ }^{23}$, R. Silvotti ${ }^{24}$, A. Piccioni ${ }^{25}$, G. Vauclair ${ }^{26}$, N. Dolez ${ }^{26}$, M. Chevreton ${ }^{27}$, J. Deetjen ${ }^{28}$, S. Dreizlere ${ }^{28,29}$, S. Schuh ${ }^{28,29}$, J. M. Gonzalez Perez ${ }^{30}$, R. Østensen ${ }^{31}$, A. Ulla ${ }^{32}$, M. Manteiga ${ }^{32}$, O. Suarez ${ }^{32}$, M. R. Burleigh ${ }^{33}$, and M. A. Barstow ${ }^{33}$

Received 20 April 2004 / Accepted 31 October 2004

\begin{abstract}
BPM 37093 is the only hydrogen-atmosphere white dwarf currently known which has sufficient mass $\left(\sim 1.1 M_{\odot}\right)$ to theoretically crystallize while still inside the ZZ Ceti instability strip $\left(T_{\text {eff }} \sim 12000 \mathrm{~K}\right)$. As a consequence, this star represents our first opportunity to test crystallization theory directly. If the core is substantially crystallized, then the inner boundary for each pulsation mode will be located at the top of the solid core rather than at the center of the star, affecting mainly the average period spacing. This is distinct from the "mode trapping" caused by the stratified surface layers, which modifies the pulsation periods more selectively. In this paper we report on Whole Earth Telescope observations of BPM 37093 obtained in 1998 and 1999. Based on a simple analysis of the average period spacing we conclude that a large fraction of the total stellar mass is likely to be crystallized.
\end{abstract}

Key words. stars: evolution - stars: individual: BPM 37093 - stars: interiors - stars: oscillations - white dwarfs

^ Based on observations obtained at: Observatório do Pico dos Dias (OPD) Brazil, the European Southern Observatory (ESO) Chile, South African Astronomical Observatory (SAAO), Mt. John University Observatory (MJUO) New Zealand, Siding Spring Observatory (SSO) Australia, and Cerro Tololo Inter-American Observatory (CTIO), a division of the National Optical Astronomy Observatories, which is operated by the Association of Universities for Research in Astronomy, Inc. under cooperative agreement with the National Science Foundation.

1 Departamento de Física Universidade Federal de Santa Catarina, CP 476, 88040-900 Florianópolis, SC, Brazil

e-mail: kanaan@astro.ufsc.br

2 Apache Point Observatory, 2001 Apache Point Road, PO Box 59, Sunspot, NM 88349, USA

3 Department of Astronomy, 1 University Station Stop C1400, University of Texas, Austin, TX 78712, USA

${ }^{4}$ Instituto de Física, Universidade Federal de Rio Grande do Sul, CP 10501, 91501-970 Porto Alegre, RS, Brazil

5 Institute of Astronomy, University of Cambridge, Madingley Road, Cambridge CB3 OHA, UK

${ }^{6}$ Harvard-Smithsonian Center for Astrophysics, USA

7 Departamento de Fisica e Quimica, UCS, Brazil

8 Dept. of Physics \& Astronomy, Iowa State University, USA

9 Astronomy Dept., Southwest Missouri State University, USA

${ }^{10}$ Dept. of Physics \& Astronomy, University of Delaware, USA
11 Southwestern University, Georgetown, TX, USA

12 South African Astronomical Observatory, South Africa

13 Victoria University of Wellington, New Zealand

14 Chatterton Astronomy Dept., University of Sydney, Australia

15 Astronomical Observatory, Academy of Sciences, PR China

16 Indian Space Research Organization, India

17 Wise Observatory, Tel-Aviv University, Israel

18 Institute of Theoretical Physics \& Astronomy, Lithuania

19 Vilnius University, Lithuania

${ }^{20}$ Mt. Suhora Observatory, Cracow Pedagogical University, Poland

21 Astronomical Observatory, Jagiellonia University, Poland

${ }^{22}$ Copernicus Astronomical Center, Poland

23 Institut für Astronomie, Universität Wien, Austria

${ }^{24}$ INAF - Osservatorio Astronomico di Capodimonte, Italy

25 Dipartimento di Astronomia, Università di Bologna, Italy

${ }^{26}$ Université Paul Sabatier, Observatoire Midi-Pyrénées, France

27 Observatoire de Paris-Meudon, France

${ }^{28}$ Institut für Astronomie und Astrophysik, Germany

${ }^{29}$ Universitätssternwarte Göttingen, Germany

${ }^{30}$ University of Troms $\varnothing$, Norway

31 Isaac Newton Group, Spain

32 Departamento de Física Aplicada, Universidad de Vigo, Spain

33 Dept. of Physics \& Astronomy, University of Leicester, UK 


\section{Introduction}

Since 1960 most astronomers have agreed that cool white dwarfs must eventually crystallize (Kirzhnitz 1960; Abrikosov 1960; Salpeter 1961). The process theoretically begins when the electrostatic interaction between the ions becomes much larger than the thermal energy. This effect is based on such well known physics that it has become widely accepted without ever having been tested empirically.

BPM 37093 is a ZZ Ceti star (Kanaan et al. 1992) with an unusually high mass $\left(1.10 M_{\odot}\right.$, Bergeron et al. 2004). White dwarfs this massive are subject to much higher pressures and densities in their cores, and we expect a $1.0 M_{\odot}$ white dwarf to begin crystallizing at temperatures within or above the ZZ Ceti instability strip (Wood 1992; Winget et al. 1997). Our goal in observing BPM 37093 with the Whole Earth Telescope (WET, Nather et al. 1990) was to obtain seismological data to determine whether or not the stellar interior is crystallized. The fundamental objective was simply to detect as many independent pulsation modes as possible, and then to compare the observed frequencies with those calculated from white dwarf models that have been artificially crystallized to various degrees.

For years we have faced a troubling ambiguity between the effects of the crystallized mass fraction $\left(M_{\mathrm{cr}}\right)$, the hydrogen layer mass $\left(M_{\mathrm{H}}\right)$, and the stellar mass $\left(M_{*}\right)$ and effective temperature $\left(T_{\text {eff }}\right)$. Changes to these four characteristics of white dwarf models can all modify the average spacing between the calculated pulsation periods (see Montgomery \& Winget 1999, Eq. (7)). Fortunately, the latter two quantities can be constrained by fitting model atmospheres to spectroscopic observations, but the others can only be determined through asteroseismology. Recent improvements in our theoretical description of the composition transition zones between the stratified surface layers in our models (Córsico et al. 2002; Althaus et al. 2003) have helped to reduce the degeneracy between $M_{\mathrm{H}}$ and $M_{\mathrm{cr}}$. However, the huge number of possible parameter combinations, and the need for an efficient method of exploring them, remained serious obstacles to progress until recently (Metcalfe \& Charbonneau 2003; Metcalfe et al. 2004).

Like the cool DAV G 29-38 (Kleinman et al. 1998), BPM 37093 exhibits irregular modulations in the amplitudes of its pulsation modes. On one occasion all of the modes vanished below the detection threshold of $\sim 1 \mathrm{mma}$ (Kanaan et al. 1998). However, the modes that disappeared were observed to reappear later with the same pulsation frequencies. This gives us confidence that we can learn more about this star by using the full set of frequencies that have been observed over time - a concept known as "time-ensemble" asteroseismology, pioneered by Kleinman et al. (1998). In this paper we report WET observations obtained in 1998 and 1999, and we use the identified pulsation periods to define a range for the average period spacing. This allows us to constrain $M_{\mathrm{H}}$ and $M_{\mathrm{cr}}$ by following the analysis of Montgomery \& Winget (1999) with an updated prescription for the envelope composition transition zones.

\section{Observations}

In 1996 and 1997, observations of BPM 37093 were obtained from the $0.9 \mathrm{~m}$ telescope at CTIO and the $1.6 \mathrm{~m}$ telescope at Observatório Pico dos Dias (OPD, Brazil) respectively. The two initial goals of these observations were: 1) to identify as many pulsation modes as possible, to help constrain asteroseismological model fitting, and 2) to find stable pulsation modes suitable for measuring the rate of period change $(\dot{P})$, as has been done for other white dwarfs (Costa et al. 1999; Kepler et al. 2000a; Mukadam et al. 2003). Further analysis (see Sect. 3) revealed that no modes were stable enough to use for $\dot{P}$ measurements. After these two attempts to obtain singlesite data on BPM 37093, it became clear that we would be unable to resolve the pulsation spectrum of this star from a single observatory. By 1997 we had already accumulated more than $100 \mathrm{~h}$ of photometry on BPM 37093, which led to the identification of only 4 pulsation modes with highly variable amplitudes (Kanaan et al. 1998).

BPM 37093 was chosen as the southern primary target for a Whole Earth Telescope campaign (XCOV 16) in 1998, and again in 1999 (XCOV 17) to coincide with simultaneous observations using the Hubble Space Telescope (HST). A journal of observations for the data obtained for these two campaigns is shown in Tables 1 and 2. Overall, we obtained more than $142 \mathrm{~h}$ of data in April-May 1998 with a duty cycle of 50\% during the central 10 days, and an impressive 180 hours in April 1999 with a better duty cycle of $65 \%$ during the central 10 days. The latter observations included almost complete coverage during the two scheduled HST visits near the middle of the campaign, and preliminary results were reported by Nitta et al. (2000).

The primary goal of the HST observations was to use the limb darkening method devised by Robinson et al. (1995) to provide an independent determination of the spherical degree $(\ell)$ for each pulsation mode. Unfortunately, this method could be applied only to the modes with the highest amplitudes, and the observations were not sensitive enough to distinguish between $\ell=1$ and $\ell=2$. In this paper we infer $\ell$ based only on the average period spacing between modes.

\section{Frequency analysis}

One of the initial goals of our observations was to identify pulsation modes stable enough to measure the rate of period change $(\dot{P})$. As a white dwarf star cools over time, the slow change in the thermal structure should lead to a detectable increase in the pulsation periods. We expect that the periods in a crystallizing star should change more slowly than in other ZZ Ceti stars because the associated release of latent heat causes the temperature to drop more slowly than in a noncrystallizing star.

However, the observations from CTIO in 1996 made it clear that $\dot{P}$ measurements would not be possible for BPM 37093. In Fig. 1 we show the evolution of daily Fourier Transforms (FTs), night by night during the 10 night run. On the tenth night, the pulsations vanished below the detection threshold. Comparing the other panels of Fig. 1, it is also clear that the amplitudes of the detected modes are highly variable. Kanaan et al. (1998) 
Table 1. Journal of observations in 1998 (XCOV 16).

\begin{tabular}{|c|c|c|c|c|}
\hline Run & Telescope & $\overline{\overline{\text { Date }}}$ & $\begin{array}{l}\text { Start } \\
\text { (UT) }\end{array}$ & $\begin{array}{r}\text { Length } \\
(\mathrm{s})\end{array}$ \\
\hline an-0069 & CTIO $1.5 \mathrm{~m}$ & 1998 Apr. 17 & $02: 42: 20$ & 23540 \\
\hline an-0070 & CTIO $1.5 \mathrm{~m}$ & 1998 Apr. 17 & $23: 30: 30$ & 37460 \\
\hline an- 0071 & CTIO $1.5 \mathrm{~m}$ & 1998 Apr. 18 & $23: 16: 00$ & 20630 \\
\hline an-0072 & CTIO $1.5 \mathrm{~m}$ & 1998 Apr. 19 & $07: 42: 20$ & 6790 \\
\hline an- 0073 & CTIO $1.5 \mathrm{~m}$ & 1998 Apr. 20 & $23: 20: 10$ & 37350 \\
\hline dj-001 & SAAO $1.9 \mathrm{~m}$ & 1998 Apr. 21 & 21:04:00 & 19400 \\
\hline an-0074 & CTIO $1.5 \mathrm{~m}$ & 1998 Apr. 21 & $23: 25: 40$ & 36770 \\
\hline dj-002 & SAAO $1.9 \mathrm{~m}$ & 1998 Apr. 22 & $17: 32: 00$ & 20150 \\
\hline an-0075 & CTIO $1.5 \mathrm{~m}$ & 1998 Apr. 22 & $23: 20: 40$ & 36860 \\
\hline ro107 & OPD $1.6 \mathrm{~m}$ & 1998 Apr. 23 & 03:57:00 & 5430 \\
\hline dj-003 & SAAO $1.9 \mathrm{~m}$ & 1998 Apr. 23 & $17: 18: 00$ & 37330 \\
\hline ro108 & OPD $1.6 \mathrm{~m}$ & 1998 Apr. 23 & $22: 22: 30$ & 4700 \\
\hline ap2498q2 & MJUO $1.0 \mathrm{~m}$ & 1998 Apr. 24 & 09:02:10 & 31110 \\
\hline $\mathrm{dj}-004$ & SAAO $1.9 \mathrm{~m}$ & 1998 Apr. 24 & $17: 29: 00$ & 37350 \\
\hline an-0076 & CTIO $1.5 \mathrm{~m}$ & 1998 Apr. 24 & $23: 43: 30$ & 35230 \\
\hline ro111 & OPD $1.6 \mathrm{~m}$ & 1998 Apr. 25 & $00: 12: 10$ & 6650 \\
\hline ap2598q1 & MJUO $1.0 \mathrm{~m}$ & 1998 Apr. 25 & $10: 20: 20$ & 6060 \\
\hline ap2598q2 & MJUO $1.0 \mathrm{~m}$ & 1998 Apr. 25 & $12: 50: 00$ & 14280 \\
\hline $\mathrm{dj}-005$ & SAAO $1.9 \mathrm{~m}$ & 1998 Apr. 25 & $17: 34: 00$ & 33580 \\
\hline ro112 & OPD $1.6 \mathrm{~m}$ & 1998 Apr. 26 & $01: 50: 30$ & 7840 \\
\hline ro113 & OPD $1.6 \mathrm{~m}$ & 1998 Apr. 26 & $04: 53: 30$ & 4210 \\
\hline ap2698q1 & MJUO $1.0 \mathrm{~m}$ & 1998 Apr. 26 & 08:06:00 & 30410 \\
\hline $\mathrm{dj}-006$ & SAAO $1.9 \mathrm{~m}$ & 1998 Apr. 26 & $17: 14: 00$ & 37880 \\
\hline an-0077 & CTIO $1.5 \mathrm{~m}$ & 1998 Apr. 27 & $03: 17: 20$ & 21770 \\
\hline ap2798q1 & MJUO $1.0 \mathrm{~m}$ & 1998 Apr. 27 & $13: 17: 30$ & 37460 \\
\hline dj-007 & SAAO $1.9 \mathrm{~m}$ & 1998 Apr. 27 & $19: 21: 00$ & 38030 \\
\hline gv-0522 & ESO $2.2 \mathrm{~m}$ & 1998 Apr. 28 & 01:01:10 & 24570 \\
\hline db9801 & SAAO $1.9 \mathrm{~m}$ & 1998 Apr. 28 & $19: 24: 00$ & 37900 \\
\hline db9802 & SAAO $1.9 \mathrm{~m}$ & 1998 Apr. 29 & $19: 14: 00$ & 32880 \\
\hline gv-0523 & ESO $2.2 \mathrm{~m}$ & 1998 Apr. 29 & $23: 21: 00$ & 21770 \\
\hline $\mathrm{db} 9803$ & SAAO $1.9 \mathrm{~m}$ & 1998 Apr. 30 & $17: 04: 50$ & 39130 \\
\hline gv-0524 & ESO $2.2 \mathrm{~m}$ & 1998 Apr. 30 & $23: 27: 00$ & 13310 \\
\hline db9804 & $\mathrm{SAAO} 1.9 \mathrm{~m}$ & 1998 Мау 01 & 21:08:00 & 31020 \\
\hline $\mathrm{db} 9805$ & SAAO $1.9 \mathrm{~m}$ & 1998 May 02 & $17: 21: 30$ & 37320 \\
\hline db9806 & SAAO $1.9 \mathrm{~m}$ & 1998 May 03 & $17: 17: 10$ & 33510 \\
\hline
\end{tabular}

demonstrated that these amplitude changes must be intrinsic to the star, rather than due to the beating of closely spaced modes, since there was no correlation between the changes in amplitude and phase. Such intrinsic behavior has been reported for other ZZ Ceti stars on a timescale of months (Kleinman et al. 1998), but not from night to night as observed in BPM 37093. These short-timescale variations will lead us to derive average amplitudes from the FTs of long data sets, but will still allow us to identify the pulsation modes which have the highest amplitudes and the longest lifetimes.

The FTs of the two WET campaigns on BPM 37093 are shown in Fig. 2. In each panel we have marked all of the peaks that exceed 4 times the observational noise level. During XCOV 16 we detected many more modes than were evident in the 1996 observations - of the 8 frequencies detected, only 2 coincide with those seen previously. Comparing this with XCOV 17, it seems clear that the detection of more independent modes in XCOV 16 can be attributed to the star itself, rather than to differences in our detection threshold. During the second WET campaign on BPM 37093 we detected
Table 2. Journal of observations in 1999 (XCOV 17).

\begin{tabular}{|c|c|c|c|c|}
\hline Run & Telescope & $\overline{\text { Date }}$ & $\begin{array}{l}\text { Start } \\
\text { (UT) }\end{array}$ & $\begin{array}{r}\text { Length } \\
\text { (s) }\end{array}$ \\
\hline saccd001 & SAAO $0.75 \mathrm{~m}$ & 1999 Apr. 06 & $23: 43: 00$ & 10680 \\
\hline saccd002 & SAAO $0.75 \mathrm{~m}$ & 1999 Apr. 07 & $21: 16: 33$ & 3960 \\
\hline ap0899q1 & $\mathrm{SSO} 1.0 \mathrm{~m}$ & 1999 Apr. 08 & 10:38:00 & 20130 \\
\hline ap0899q2 & $\mathrm{SSO} 1.0 \mathrm{~m}$ & 1999 Apr. 08 & $16: 34: 00$ & 3620 \\
\hline ap0899q3 & $\mathrm{SSO} 1.0 \mathrm{~m}$ & 1999 Apr. 08 & $17: 52: 30$ & 5460 \\
\hline ap0999q1 & $\mathrm{SSO} 1.0 \mathrm{~m}$ & 1999 Apr. 09 & $10: 15: 20$ & 29000 \\
\hline tsm-0033 & CTIO $1.5 \mathrm{~m}$ & 1999 Apr. 10 & 02:10:00 & 26100 \\
\hline ap1099q1 & $\mathrm{SSO} 1.0 \mathrm{~m}$ & 1999 Apr. 10 & 09:04:40 & 36680 \\
\hline saccd003 & SAAO $0.75 \mathrm{~m}$ & 1999 Apr. 10 & 18:00:00 & 22060 \\
\hline tsm-0035 & CTIO $1.5 \mathrm{~m}$ & 1999 Apr. 11 & 05:55:00 & 6620 \\
\hline ap1199q1 & $\mathrm{SSO} 1.0 \mathrm{~m}$ & 1999 Apr. 11 & 08:59:50 & 37110 \\
\hline saccd004 & SAAO $0.75 \mathrm{~m}$ & 1999 Apr. 11 & 18:09:00 & 33300 \\
\hline ap1299q1 & $\mathrm{SSO} 1.0 \mathrm{~m}$ & 1999 Apr. 12 & 11:03:00 & 29900 \\
\hline saccd005 & SAAO $0.75 \mathrm{~m}$ & 1999 Apr. 12 & $18: 18: 30$ & 32700 \\
\hline tsm-0042 & CTIO $1.5 \mathrm{~m}$ & 1999 Apr. 13 & 00:43:00 & 31440 \\
\hline ap1399q1 & $\mathrm{SSO} 1.0 \mathrm{~m}$ & 1999 Apr. 13 & 09:35:00 & 9300 \\
\hline ap1399q2 & $\mathrm{SSO} 1.0 \mathrm{~m}$ & 1999 Apr. 13 & $12: 29: 00$ & 25000 \\
\hline saccd006 & SAAO $0.75 \mathrm{~m}$ & 1999 Apr. 13 & $18: 17: 20$ & 16920 \\
\hline tsm-0043 & CTIO $1.5 \mathrm{~m}$ & 1999 Apr. 13 & 23:48:00 & 34410 \\
\hline ap1499q1 & $\mathrm{SSO} 1.0 \mathrm{~m}$ & 1999 Apr. 14 & 09:14:00 & 36700 \\
\hline saccd007 & SAAO $0.75 \mathrm{~m}$ & 1999 Apr. 14 & $19: 33: 25$ & 21840 \\
\hline tsm-0044 & CTIO $1.5 \mathrm{~m}$ & 1999 Apr. 14 & $23: 42: 00$ & 34560 \\
\hline saccd008 & SAAO $0.75 \mathrm{~m}$ & 1999 Apr. 15 & $17: 23: 45$ & 20250 \\
\hline tsm-0045 & CTIO $1.5 \mathrm{~m}$ & 1999 Apr. 16 & 00:08:30 & 32730 \\
\hline tsm-0046 & CTIO $1.5 \mathrm{~m}$ & 1999 Apr. 17 & 00:10:40 & 32100 \\
\hline ap1799q1 & MJUO $1.0 \mathrm{~m}$ & 1999 Apr. 17 & $09: 34: 30$ & 7970 \\
\hline saccd009 & SAAO $0.75 \mathrm{~m}$ & 1999 Apr. 17 & $17: 17: 40$ & 32220 \\
\hline ita180499 & OPD $0.6 \mathrm{~m}$ & 1999 Apr. 18 & $03: 49: 40$ & 11000 \\
\hline tsm-0047 & CTIO $1.5 \mathrm{~m}$ & 1999 Apr. 18 & 04:46:00 & 15640 \\
\hline ap1899q1 & MJUO $1.0 \mathrm{~m}$ & 1999 Apr. 18 & 07:30:00 & 7690 \\
\hline ap1899q2 & MJUO $1.0 \mathrm{~m}$ & 1999 Apr. 18 & $11: 36: 00$ & 8310 \\
\hline ap1899q3 & MJUO $1.0 \mathrm{~m}$ & 1999 Apr. 18 & $14: 04: 30$ & 14400 \\
\hline tsm-0048 & CTIO $1.5 \mathrm{~m}$ & 1999 Apr. 19 & 00:04:30 & 32330 \\
\hline ap1999q1 & MJUO $1.0 \mathrm{~m}$ & 1999 Apr. 19 & 08:04:20 & 6950 \\
\hline ap1999q4 & MJUO $1.0 \mathrm{~m}$ & 1999 Apr. 19 & $15: 05: 50$ & 10500 \\
\hline ita190499 & OPD $0.6 \mathrm{~m}$ & 1999 Apr. 19 & $22: 15: 10$ & 32700 \\
\hline ro123 & OPD $1.6 \mathrm{~m}$ & 1999 Apr. 20 & $01: 23: 40$ & 18800 \\
\hline ita200499 & OPD $0.6 \mathrm{~m}$ & 1999 Apr. 20 & $03: 33: 24$ & 8420 \\
\hline ita210499 & OPD $0.6 \mathrm{~m}$ & 1999 Apr. 21 & $22: 15: 07$ & 18080 \\
\hline
\end{tabular}

7 frequencies, of which 4 coincide with others seen previously. Only the $512 \mathrm{~s}$ and $661 \mathrm{~s}$ modes are unique to the data obtained for XCOV 17. The full list of frequencies detected in these two WET campaigns is shown in Table 3, along with the mode identifications of Metcalfe et al. (2004) $)^{34}$ for those modes found in the preliminary analysis of Kanaan et al. (2000).

We can understand the erratic behavior of BPM 37093 by considering previous observations of other ZZ Ceti pulsators. Kleinman et al. (1998) documented very similar results for the star G 29-38, and proposed that each set of observations can provide a subset of the full spectrum of normal modes. The fundamental idea behind this hypothesis is that the underlying structure of the star does not change on the timescales of the observed amplitude variations. Instead, something in the excitation mechanism must select certain modes and exclude others

${ }^{34}$ For the $582 \mathrm{~s}$ mode, we adopt the $\ell=2$ identification. 


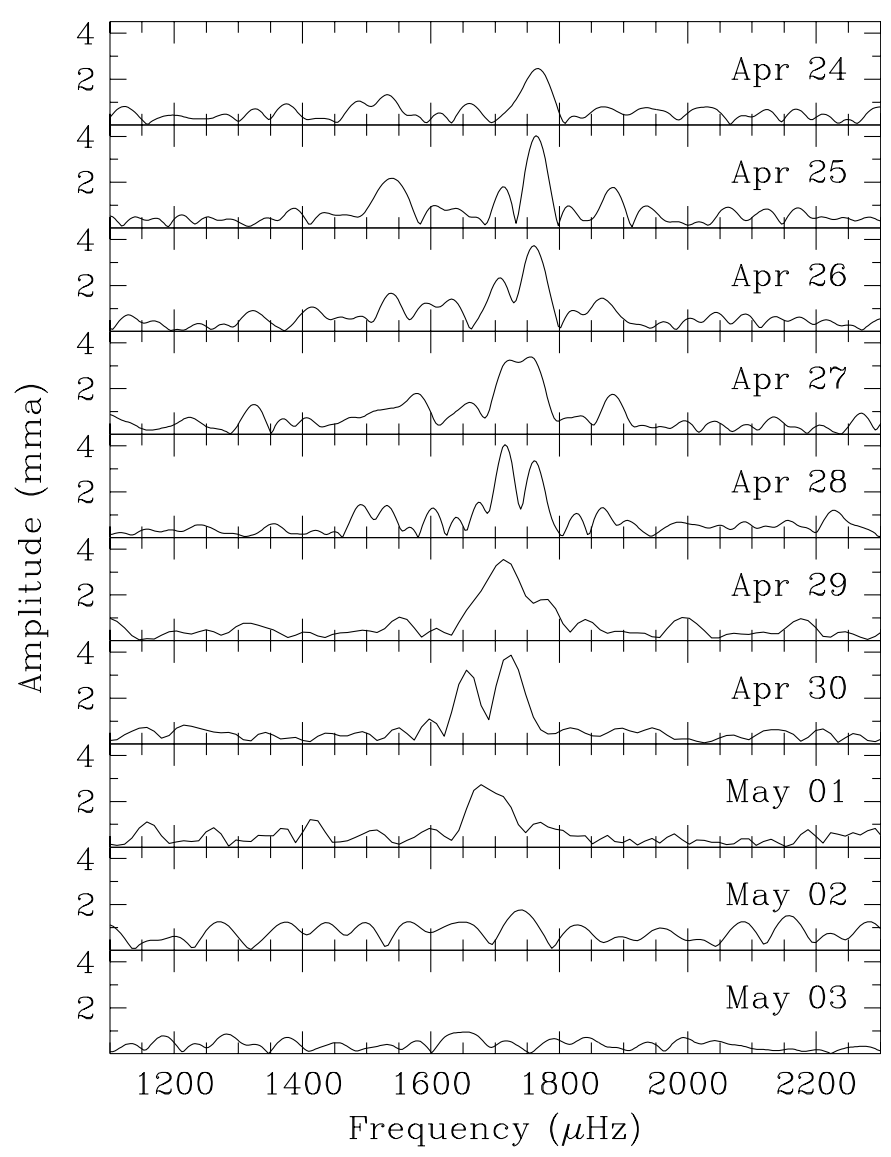

Fig. 1. Nightly FTs of BPM 37093 during ten nights of observations from CTIO in 1996. The amplitudes are highly variable on short timescales. All of the modes vanish below the detection threshold on the final night.

in a manner that varies over time. Setting aside the question of what specifically causes these amplitude modulations, we only need to assume that the observed frequencies are, in each case, normal modes of oscillation that can be described by spherical harmonics. Such an assumption rests on a firm body of evidence (Robinson et al. 1982; Kepler et al. 2000b; Clemens et al. 2000), and is also supported by the fact that modes which disappear below the detection threshold are observed to reappear later with the same frequencies.

\section{Interpretation}

We adopt for our analysis the full set of frequencies that have been observed in BPM 37093 over time, giving preference to the WET campaigns for their superior frequency precision. For the isolated frequencies we assume that each mode has an azimuthal order $m=0$ (see Metcalfe 2003), and for the modes consisting of two closely-spaced frequencies we use the average of the two. For modes that were observed in both campaigns, we use the frequency from the observation with the highest amplitude. This leads to a total of 7 independent modes that have been identified as $\ell=2$ by Metcalfe et al. (2004). The average period spacing between consecutive radial overtones for these 7 modes is $\langle\Delta P\rangle=17.6 \pm 1.1 \mathrm{~s}$. This implies that the $\ell=2$ identification should be correct for the majority
Table 3. Frequencies detected from two WET campaigns.

\begin{tabular}{|c|c|c|c|c|c|c|}
\hline \multirow[t]{2}{*}{$\overline{\mathrm{XCOV}}$} & \multirow{2}{*}{$\begin{array}{c}\text { Frequency } \\
(\mu \mathrm{Hz})\end{array}$} & \multirow{2}{*}{$\begin{array}{c}\text { Period } \\
(\mathrm{s})\end{array}$} & \multirow{2}{*}{ 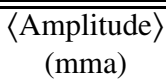 } & \multicolumn{3}{|c|}{ Identification } \\
\hline & & & & $k$ & $\ell$ & $m$ \\
\hline 17 & 1513.2 & 660.8 & 0.475 & & & \\
\hline [ 17 & 1569.5 & 637.2 & 0.650 & & & \\
\hline 16 & 1570.6 & 636.7 & 1.741 & 34 & 2 & -1 \\
\hline 17 & 1578.5 & 633.5 & 1.310 & 34 & 2 & +1 \\
\hline L 16 & 1579.2 & 633.2 & 1.081 & & & \\
\hline 16 & 1629.9 & 613.5 & 1.131 & 18 & 1 & 0 \\
\hline 16 & 1664.9 & 600.7 & 0.875 & 32 & 2 & 0 \\
\hline 16 & 1718.2 & 582.0 & 1.032 & 31 & 2 & 0 \\
\hline 17 & 1767.1 & 565.9 & 0.458 & & & \\
\hline 16 & 1768.5 & 565.5 & 1.174 & 30 & 2 & -1 \\
\hline 16 & 1777.6 & 562.6 & 0.879 & 30 & 2 & +1 \\
\hline 17 & 1820.8 & 549.2 & 0.801 & & & \\
\hline 16 & 1823.5 & 548.4 & 1.149 & 29 & 2 & 0 \\
\hline 17 & 1882.9 & 531.1 & 1.156 & 28 & 2 & 0 \\
\hline 17 & 1954.1 & 511.7 & 0.679 & 27 & 2 & 0 \\
\hline
\end{tabular}

of the modes, since an $\ell=1$ identification would yield a much larger period spacing $(\langle\Delta P\rangle \sim 30 \mathrm{~s}$, Montgomery \& Winget 1999).

Montgomery \& Winget (1999) performed a detailed study of the various model parameters that could affect the average period spacing for $\ell=2$ modes. They defined a scaling relation for $\langle\Delta P\rangle$ which had contributions from variations to four parameters, including the stellar mass and effective temperature $\left(M_{*}, T_{\text {eff }}\right)$ and the hydrogen layer mass and crystallized mass fraction $\left(M_{\mathrm{H}}, M_{\mathrm{cr}}\right)$. Fortunately, $M_{*}$ and $T_{\text {eff }}$ can both be constrained by spectral profile fitting. The most recent estimates for BPM 37093 come from Bergeron et al. (2004), who found $M_{*}=1.10 M_{\odot}$ and $T_{\text {eff }}=11730 \mathrm{~K}$. The values of the other parameters can only be determined through asteroseismology, but Montgomery \& Winget (1999) found a troubling degeneracy between $M_{\mathrm{H}}$ and $M_{\mathrm{cr}}$ that could not be broken using only the observed $\langle\Delta P\rangle$.

To get beyond the degeneracy, we need to use the individual pulsation periods in addition to $\langle\Delta P\rangle$. Fundamentally, this is possible because variations to $M_{\mathrm{H}}$ change the individual periods through "mode trapping", while variations to $M_{\text {cr }}$ affect mainly the average period spacing. If BPM 37093 is partially crystallized, the inner boundary for each pulsation mode will be located at the top of the solid core rather than at the center of the star. This reduces the size of the resonant cavity, increasing the average period spacing and modifying the periods of all of the modes. By contrast, the hydrogen layer produces a sharp chemical gradient somewhere near the surface. Any individual mode whose eigenfunction is large in this region can interact with the chemical gradient and become "trapped" - its period will be shifted more than the periods of other modes. Clearly, each mode will be modified in a different manner through this interaction.

Montgomery \& Winget (1999) understood that the signatures of $M_{\mathrm{H}}$ and $M_{\mathrm{cr}}$ are in principle distinct. However, by focusing only on the average period spacing they were unable to place strong constraints on either $M_{\mathrm{H}}$ or $M_{\mathrm{cr}}$. Our present models are somewhat improved: Montgomery \& Winget used 


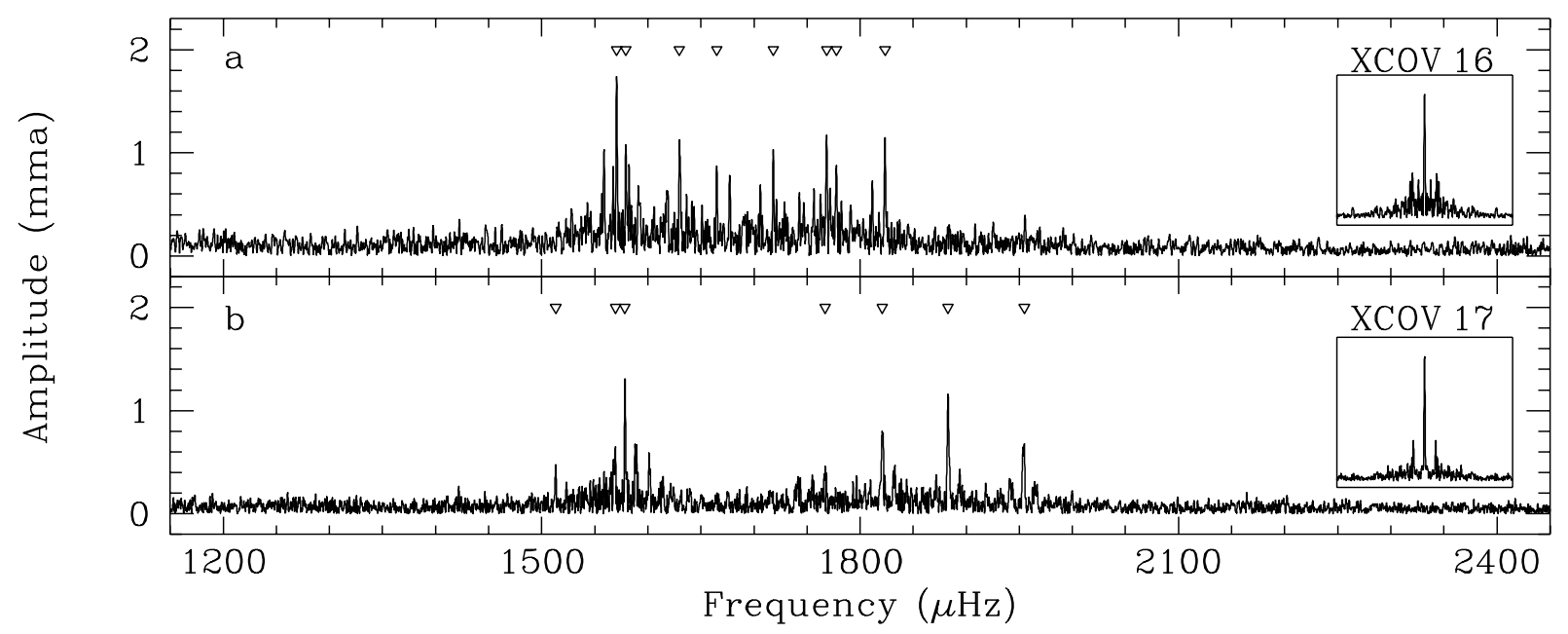

Fig. 2. Fourier Transforms and window functions at the same scale for the Whole Earth Telescope observations of the ZZ Ceti star BPM 37093 obtained during a) the XCOV 16 campaign in 1998, and b) the XCOV 17 campaign in 1999.

hydrogen profiles that were derived assuming diffusive equilibrium in the trace element approximation. This produced unrealistically sharp chemical gradients at the base of the hydrogen layer, leading to stronger mode trapping in their models. This was demonstrated by Córsico et al. (2002), who compared models that assumed diffusive equilibrium in the trace element approximation with models that computed the abundance profiles based on time-dependent diffusion calculations. In a recent extension of this work to massive ZZ Ceti stars, Althaus et al. (2003) described an improved method of calculating diffusive equilibrium profiles that compare favorably with the fully timedependent results (see their Fig. 18). We have incorporated this method of computing the hydrogen abundance profiles into the code used by Montgomery \& Winget (1999). However, since the sharpness of the hydrogen transition zone should mainly affect the mode trapping properties of the models, we expect that our new average period spacings will differ only slightly from those computed by Montgomery \& Winget (1999).

As a simple illustration of the potential of our observations, we calculated $\langle\Delta P\rangle$ for a small grid with various combinations of $M_{\mathrm{H}}$ and $M_{\mathrm{cr}}$. We fixed the mass, temperature, and helium layer thickness to the values used for Fig. 10b of Montgomery $\&$ Winget (1999), but we assumed a uniform O core. We show this grid of models in Fig. 3 with the shaded $1 \sigma$ range of the average period spacing from the WET observations of BPM 37093. As expected, the average period spacing of the $0 \%$ crystallized model is virtually identical to that found by Montgomery \& Winget (1999). However, due to the different assumed C/O profiles, the crystallized curves have shifted with respect to the results of Montgomery \& Winget (1999).

Unfortunately, the degeneracy between $M_{\mathrm{H}}$ and $M_{\mathrm{cr}}$ is still present, but we have not yet used the hidden third dimension of the grid: at each point we have also computed the root-meansquare differences between the observed and calculated periods $\left(\sigma_{\mathrm{P}}\right)$, to exploit the information contained in the individual modes. Although the observed range of $\langle\Delta P\rangle$ can accommodate values of $\log \left(M_{\mathrm{H}} / M_{*}\right)$ between $-5\left(M_{\mathrm{cr}}=80-90 \%\right)$ and -8 (uncrystallized), not all hydrogen layer masses are equally successful at reproducing the individual modes. The model with

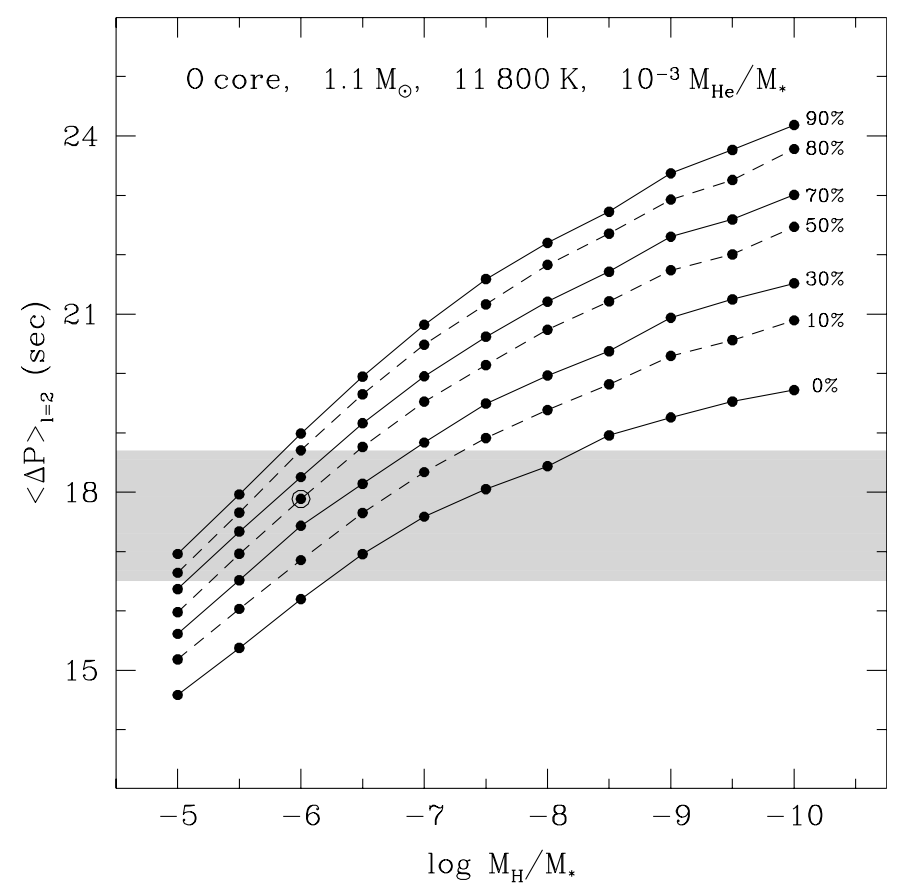

Fig. 3. The average period spacing of a small grid of models with various combinations of $M_{\mathrm{H}}$ and $M_{\mathrm{cr}}$. The $1 \sigma$ range of the observed average period spacing for BPM 37093 is shown as a shaded area, and the circled point indicates the model with the smallest rms difference between the observed and calculated periods (see text for details).

$\log \left(M_{\mathrm{H}} / M_{*}\right)=-6$ and $M_{\mathrm{cr}}=50 \%$ has $\sigma_{\mathrm{P}}=1.08 \mathrm{~s}$, which is substantially better than anything else in this small grid (the next best model has $\sigma_{\mathrm{P}}=1.70 \mathrm{~s}$ ). A theoretical model with this same set of structural parameters is expected to be between 66-92\% crystallized for a C/O mixture, or even more crystallized if the core is composed of an $\mathrm{O} / \mathrm{Ne}$ mixture (see Córsico et al. 2004, for some recent calculations).

Of course, the individual modes also contain information about the mass, temperature, helium layer, and core composition. If we have fixed these parameters incorrectly, it is likely 
that we have found a locally optimal match to the observed periods rather than the global solution. Montgomery \& Winget (1999) recognized this difficulty, and discussed the need for an automated procedure to search this enormous parameter-space. Such a procedure, based on a parallel genetic algorithm, has recently been developed (Metcalfe \& Charbonneau 2003) and applied to this problem. The initial results from this large-scale exploration of the models have been published separately by Metcalfe et al. (2004), who present a more detailed discussion of the initial model fitting results.

\section{Discussion}

The Whole Earth Telescope has once again deciphered the complex pulsation spectrum of an astrophysically interesting white dwarf star. BPM 37093 is the only known ZZ Ceti star massive enough to allow a seismological test of crystallization theory, and previous attempts to understand it from single site observations were not successful. The superior frequency precision and extended coverage of two WET campaigns finally allowed us to document a series of 9 independent pulsation modes in this star. While it is always useful to search for additional frequencies to help constrain the model fitting, the observational requirements of this project have now largely been satisfied.

The limiting factor in our ability to test crystallization theory through asteroseismology of BPM 37093 is now computational. The initial study of Metcalfe et al. (2004) was limited to several fixed masses and core compositions, but all of their optimal models suggested that a large fraction of the core is crystallized - a result that is qualitatively supported by our simple analysis of the average period spacing. We have only fit $M_{\mathrm{cr}}$ to the nearest $0.1 M_{*}$, but Montgomery \& Winget (1999) showed that the pulsations are sensitive to changes of $0.01 M_{*}$ in this parameter. As computers get faster, we should be able to extend the genetic algorithm based model fitting method to treat $M_{\mathrm{cr}}, M_{*}$ and the composition of the liquid portion of the core as fully adjustable parameters. In the meantime, we should apply the same fitting procedure to lower mass ZZ Ceti stars that are not expected to be crystallized, as a check of the method.

The Sloan Digital Sky Survey (SDSS) has recently discovered several new ZZ Ceti stars that may also be massive enough to test crystallization theory (Mukadam et al. 2004). All of the new pulsators in the SDSS sample are significantly fainter than the previously known white dwarfs, so larger telescopes will probably be required to resolve their pulsation spectra. Several current and planned space missions (MOST, Walker et al. 2003; COROT, Baglin et al. 2002) promise to revolutionize the field of asteroseismology in the coming decade. However, none of them is likely to contribute to our understanding of pulsating white dwarfs stars, which are too faint to be observed by these satellites. The Whole Earth Telescope remains the instrument of choice for these fainter targets. The availability of larger ground-based telescopes may allow the WET concept to be extended to $2 \mathrm{~m}$ and $4 \mathrm{~m}$ class instruments. With a growing harvest of new objects from SDSS, the future looks bright for white dwarf asteroseismology.

Acknowledgements. This work was partially supported by NASA, NSF, Brazilian institutions FAPERGS, CNPq, CAPES, and FINEP, and by the Smithsonian Institution through a CfA Postdoctoral Fellowship. PM is partially supported by Polish KBN grants 5 P03D 01220 and 5 P03D 03020.

\section{References}

Abrikosov, A. 1960, Zh. Eksp. Teor. Fiz., 39, 1798

Althaus, L. G., Serenelli, A. M., Córsico, A. H., \& Montgomery, M. H. 2003, A\&A, 404, 593

Baglin, A., Auvergne, M., Catala, C., et al. 2002, in ASP Conf. Ser., 259, IAU Coll., 185, Radial and Nonradial Pulsationsn as Probes of Stellar Physics, 626

Bergeron, P., Fontaine, G., Billères, M., Boudreault, S., \& Green, E. M. 2004, ApJ, 600, 404

Córsico, A. H., Benvenuto, O. G., Althaus, L. G., \& Serenelli, A. M. 2002, MNRAS, 332, 392

Córsico, A. H., García-Berro, E., Althaus, L. G., \& Isern, J. 2004, A\&A, 427, 923

Clemens, J. C., van Kerkwijk, M. H., \& Wu, Y. 2000, MNRAS, 314, 220

Costa, J. E. S., Kepler, S. O., \& Winget, D. E. 1999, ApJ, 522, 973

Kanaan, A., Kepler, S. O., Giovannini, O., \& Diaz, M. 1992, ApJ, 390, L89

Kanaan, A., Kepler, S. O., Giovannini, O., et al. 1998, Baltic Astron., 7,183

Kanaan, A., Nitta-Kleinman, A., Winget, D. E., et al. 2000, Baltic Astron., 9, 87

Kepler, S. O., Mukadam, A., Winget, D. E., et al. 2000a, ApJ, 534, L185

Kepler, S. O., Robinson, E. L., Koester, D., et al. 2000b, ApJ, 539, 379

Kirzhnitz, D. A. 1960, Soviet Phys. - JETP, 11, 365

Kleinman, S. J., Nather, R. E., Winget, D. E., et al. 1998, ApJ, 495, 424

Metcalfe, T. S. 2003, Baltic Astron., 12, 247

Metcalfe, T. S., \& Charbonneau, P. 2003, J. Comput. Phys., 185, 176

Metcalfe, T. S., Montgomery, M. H., \& Kanaan, A. 2004, ApJ, 605, L133

Montgomery, M. H., \& Winget, D. E. 1999, ApJ, 526, 976

Mukadam, A. S., Kepler, S. O., Winget, D. E., et al. 2003, ApJ, 594, 961

Mukadam, A. S., Mullally, F., Nather, R. E., et al. 2004, ApJ, 607, 982 Nather, R. E., Winget, D. E., Clemens, J. C., Hansen, C. J., \& Hine, B. P. 1990, ApJ, 361, 309

Nitta, A., Kanaan, A., Kepler, S. O., et al. 2000, Baltic Astron., 9, 97 Robinson, E. L., Kepler, S. O., \& Nather, R. E. 1982, ApJ, 259, 219

Robinson, E. L., Mailloux, T. M., Zhang, E., et al. 1995, ApJ, 438, 908

Salpeter, E. E. 1961, ApJ, 134, 669

Walker, G., Matthews, J., Kuschnig, R., et al. 2003, PASP, 115, 1023

Winget, D. E., Kepler, S. O., Kanaan, A., Montgomery, M. H., \& Giovannini, O. 1997, ApJ, 487, L191

Wood, M. A. 1992, ApJ, 386, 539 